\title{
A theoretical analysis of trading rules: an application to the moving average case with Markovian returns
}

\author{
EMMANUEL ACAR ${ }^{1}$ and STEPHEN E. SATCHELL ${ }^{2 *}$ \\ ${ }^{1}$ Banque Nationale de Paris plc, London, UK and ${ }^{2}$ Trinity College, Cambridge and Faculty of Economics, \\ University of Cambridge, Cambridge, UK
}

Received September 1995 and accepted February 1997

A general framework for analysing trading rules is presented. We discuss different return concepts and different statistical processes for returns. We then concentrate on moving average trading rules and show, in the case of moving average models of length two, closed form expressions for the characteristic function of realized returns when the underlying return process follows a switching Markovian Gaussian process. An example is included which illustrates the technique.

Keywords: moving averages, switching Markov models, trading rules

\section{Introduction}

The purpose of this article is to examine the statistical properties of one of the most popular technical trading rules: the moving average method. The basic assumption of technical analysis is that 'everything is in the rate'. Then if markets move in trends, defining the prevailing trend and being able to identify early reversals through forecasting methods is certainly helpful in assessing future rate developments. Forecasting techniques which use only past prices to forecast future prices are called technical indicators. They can be classified in two categories: chartism and mechanical system.

Charting is the oldest branch of technical analysis. Chartism is based on the assumption that trends and patterns in charts reflect not only all available information but the psychology of the investor as well. Analysts who use charts look for graphical cycles and repetition of patterns to discern trends. The rules derived from the analysis of charts are often subjective and, as such, chartism is considered more of an art than a science. This is primarily why it has not been possible to define chart patterns with mathematical rigour. Neftci (1991) demonstrates this point for at least two popular charts methods. He argues that they are ill-conceived and that no proper study of their usefulness can be achieved. This is why chartist techniques will be ignored in our paper, which will concentrate instead on objective rules only.

\footnotetext{
* Dr. Satchell would like to thank Inquire and the Newton Trust for Financial Support.
} 
Mechanical systems are conceived in a way to trigger indisputable sell and buy signals following a decision rule based on past data, usually by calculating if the price is above or below a particular entry point. These systems are typically not concerned with how much the price is above or below the entry point. They attempt to predict the direction of the future price without searching to forecast its level. They are used to detect major downturns and upturns of the market. The appropriateness of these indicators is conditional to the fact that trends in prices tend to persist for some time and can be detected. Three main features characterize mechanical systems: pathdependency, convexity, and non-uniqueness. By design, mechanical systems depend on the history of price movements prior to the end of the investment horizon. Consequently, they are highly pathdependent strategies. The usual rule is to trade with the trend. The trader initiates a position early in the trend and maintains that position as long as the trend continues. Almost all mechanical systems are trend-following and so exhibit convex payoffs. There are a few which belong to the family of contrary opinion indicators, known as well as reverse trend-following rules, and so display concave payoffs. They can be used on their own but usually they are applied in combination with trendfollowing systems. The main difficulty with mechanical trading systems is that a rule has to be chosen from an infinite number of alternatives. Since those systems are assumed to reflect (mechanically) the expectations of the forecaster, there can exist as many rules as there are different expectations.

There are so many relevant trading rules that it is unrealistic to list them all. In what follows we concentrate on the most popular rule among practitioners and academics: the moving average method. The use of this method to forecast financial markets goes back at least to Donchian (1957) and has since been widely used by market practitioners, as witness numerous and regular papers in Technical Analysis of Stock and Commodities and Futures. In comparison, the study of technical analysis by academics is relatively new. One of the first studies is due to Alexander (1961). Further empirical research on the usefulness of technical analysis has followed. Applications among others can be found in Goodhart and Curcio (1992) for the foreign exchange markets, in Silber (1994) for the futures markets and in Corrado and Lee (1992) for the stock markets. Recent work by LeBaron (1991, 1992), Neftci (1991), Taylor (1994), Brock et al. (1992), Levich and Thomas (1993) and Blume et al. (1994), have stressed the statistical properties of technical trading rules and the insights they might give us about the underlying process. Although Neftci (1991) has examined the Markovian properties of trading strategies, there is only a scattered literature on their analytic properties. One exception is the thesis by Acar (1993) which studies the problem in a systematic way. Broadly his results hinge on the following three ideas:

(i) the distribution of returns,

(ii) the nature of the trading rule,

(iii) the particular returns concept involved.

Given particular choices for each of these, he proves various theorems. The contribution of this paper is to present and generalize some of these results, in particular, we concentrate on data generated by a Markovian switching model, popularized and applied to asset prices by Hamilton (1989). In Section 2 we list our choices for asset return distribution, trading rules, and returns concept. In Section 3, we establish the realized rate of return for the simple moving average of order 2 under Markovian assumptions, and present an application of our results to FTSE 100 Futures returns in Section 4. Section 5 concludes the paper. 


\section{Definitions for rules and returns}

Suppose that we observe over $T$ periods the price of an asset $P_{\mathrm{t}}$ that holds for one time period. Let $X_{\mathrm{t}}=\ln \left(P_{\mathrm{t}}\right)-\ln \left(P_{\mathrm{t}-1}\right)$. Our choice of distribution for $P_{\mathrm{t}}$ or $X_{\mathrm{t}}$ is

$$
X_{\mathrm{t}}=\alpha+\varepsilon_{\mathrm{t}}
$$

where $\varepsilon_{\mathrm{t}} \sim \operatorname{iid}\left(0, \sigma^{2}\right)$, the $\log$ random walk with drift.

$$
P_{\mathrm{t}}=P_{\mathrm{t}-1}+\alpha+\varepsilon_{\mathrm{t}}
$$

where $\varepsilon_{\mathrm{t}} \sim \operatorname{iid}\left(0, \sigma^{2}\right)$, the (arithmetic) random walk with drift for prices.

A third choice of asset price distribution is a Markovian process. One example of this is the switching Markov model popularized by Hamilton (1989)

$$
\text { i.e. } X_{\mathrm{t}}=\alpha_{0}+\alpha_{1} S_{\mathrm{t}}+\varepsilon_{\mathrm{t}}
$$

where $\varepsilon_{\mathrm{t}} \sim \operatorname{iid}\left(0, \sigma^{2}\right)$ and $S_{\mathrm{t}}$ is a 2 state Markov variable, $\operatorname{Prob}\left(S_{\mathrm{t}}=1 \mid S_{\mathrm{t}-1}=1\right)=p$, and $\operatorname{Prob}\left(S_{\mathrm{t}}=0 \mid S_{\mathrm{t}-1}=0\right)=q$. This is a very simple version, we could have $\sigma$ depending upon the state, $S_{\mathrm{t}}$. The point here is that there may be high and low state returns and it may be possible for trading rules to incorporate information which involves (Markovian) information about the next state. Another example is the autoregressive (mean-reverting) process.

$$
X_{\mathrm{t}}=\beta+\alpha X_{\mathrm{t}-1}+\varepsilon_{\mathrm{t}}
$$

Turning now to trading rules, we shall restrict ourselves to the two following families, the arithmetic and geometric moving average rules.

Specifically, we shall define a variable $I_{\mathrm{t}}=1$ (buy) and $I_{\mathrm{t}}=-1$ (sell) whose values are governed by some rule, i.e. $I_{\mathrm{t}}=1$ if $f\left(X_{\mathrm{t}}, X_{\mathrm{t}-1} \ldots\right) \geqslant 0$. With this convention we define the following.

\subsection{Moving average rules}

Geometric Moving Average Rules of length $m$ are defined by the rule,

$$
I_{\mathrm{t}}=1 \quad \text { iff } \quad P_{\mathrm{t}} \geqslant\left(\prod_{j=0}^{m-1} P_{\mathrm{t}-\mathrm{j}}\right)^{\frac{1}{m}} \quad I_{\mathrm{t}}=-1 \quad \text { otherwise }
$$

Arithmetic Moving Average Rules of length $m$ are defined by

$$
I_{\mathrm{t}}=1 \quad \text { iff } \quad P_{\mathrm{t}} \geqslant \sum_{j=0}^{m-1} P_{\mathrm{t}-\mathrm{j}} / m \quad I_{\mathrm{t}}=-1 \quad \text { otherwise }
$$

If we take (natural) logarithms of (2.4), and rearrange we see that

$$
X_{\mathrm{t}} \geqslant-\sum_{j=1}^{(m-2)} \frac{(m-(j+1)) X_{\mathrm{t}-\mathrm{j}}}{(m+1)}
$$

so that a geometric MA rule in prices has a weighted arithmetic MA analogue in returns. 
Acar (1993: 61) shows that if we rearrange Equation 2.6 by $\left(1-P_{\mathrm{t}-1} / P_{\mathrm{t}}\right)+\left(1-P_{\mathrm{t}-2} / P_{\mathrm{t}}\right)+$ $\ldots\left(1-\left(P_{\mathrm{t}-\mathrm{m}+1}\right) / P_{\mathrm{t}}\right) \geqslant 0 \quad$ and $\quad$ use $\quad 1-\left(P_{\mathrm{t}-\mathrm{j}} / P_{\mathrm{t}}\right) \approx \ln \left(P_{\mathrm{t}} / P_{\mathrm{t}-\mathrm{j}}\right)=\ln \left(\left(P_{\mathrm{t}} / P_{\mathrm{t}-1}\right) \cdot\left(P_{\mathrm{t}-1} / P_{\mathrm{t}-2}\right) \ldots\right.$ $\left.\left(P_{\mathrm{t}-(\mathrm{j}-1)} / P_{\mathrm{t}-\mathrm{j}}\right)\right)=-\sum_{k=0}^{j-1} X_{\mathrm{t}-\mathrm{k}}$ then Equation 2.6 becomes, upon simplification, $\sum_{j=1}^{m-1}(m-$ j) $X_{\mathrm{t}-\mathrm{j}+1} \geqslant 0$ which is just a rearrangement of Equation 2.7. Thus we can see that the two families of rules are approximately the same assuming the near equality of arithmetic and geometric returns. Acar (1993: 3.20) provides some numerical results which show the essential equivalence of the two rules for numbers normally encountered in empirical finance. The reason we have laboured this point is that for the calculation of analytical results, if we assume log-normal prices and normal (log) rates of returns then it is more tractable to work with Equation 2.6 rather than Equation 2.7.

In the family of MA( ) rules, it turns out that the MA(2) rule leads to analytic results. Another tractable rule, and one that has practical relevance is the $\operatorname{MA}(\infty)$ rule. Many rules examined by Brock et al. (1992) are of the form involving MA(150) or MA(200)'s. Clearly if the process is stationary one can work with the asymptotic distribution. In the case of a geometric MA(2) rule, $I_{\mathrm{t}}=1$ iff $P_{\mathrm{t}} \geqslant\left(P_{\mathrm{t}} P_{\mathrm{t}-1}\right)^{1 / 2}$ iff $P_{\mathrm{t}} \geqslant P_{\mathrm{t}-1}$, iff $X_{\mathrm{t}}>0$ while for an arithmetic MA(2) rule $I_{\mathrm{t}}=1$ iff $P_{\mathrm{t}} \geqslant\left(P_{\mathrm{t}}+P_{\mathrm{t}-1}\right) / 2$ iff $P_{\mathrm{t}} \geqslant P_{\mathrm{t}-1}$ iff $X_{\mathrm{t}}>0$ so that geometric and arithmetic MA(2) rules in prices coincide and correspond to $X_{\mathrm{t}}>0$. An extension to MA rules is an MA rule with band

$$
\begin{array}{ll}
\text { If } w_{\mathrm{t}}^{(n)}=X_{\mathrm{t}}-\sum_{i=1}^{n} X_{\mathrm{t}-\mathrm{i}} / n>\beta & \text { buy, } I_{\mathrm{t}}=1 \\
\text { If } w_{\mathrm{t}}^{(n)}<-\beta & \text { sell, } I_{\mathrm{t}}=-1 \\
\text { If }-\beta<w_{\mathrm{t}}^{(n)}<\beta & \text { hold }
\end{array}
$$

Finally we deal with the issue of returns, the first concept is the standard notion of returns for a fixed period, we define this as $R_{\mathrm{N}}$ where

$$
R_{\mathrm{N}}=\sum_{j=1}^{N} X_{\mathrm{t}+\mathrm{j}} I_{\mathrm{t}+\mathrm{j}-1}
$$

For this concept to be appropriate, the investor has decided to follow the strategy for $N$ periods irrespective of how prices behave. We call this the unrealized return.

For the second concept we wish to capture the idea that if prices fall at time $n$, we will sell in time $n$. In this formulation $n$ is the value of a random variable $N$ and we call such returns realized returns, denoted by $R R_{n}$

$$
R R_{n}=\sum_{j=1}^{n} X_{j}
$$

In our definition of $R R_{n}$, if our rule is a buy and hold rule until prices fall, then $X_{1}>0, \ldots, X_{n-1}>0$, $X_{n}<0$, and $I_{t}=1, t=1, \ldots, n-1$, if $n>1, I_{n}=-1$. In the above case we assume the decision to buy is made prior to observing prices.

Because $R R_{n}$ depends upon a stochastic $n$ we need to consider the unconditional realized returns $R R$, where $R R$ is defined by,

$$
R R=\sum_{n=2}^{\infty} R R_{n} \operatorname{Prob}(N=n)
$$


and $N$ is defined by the length of the buy (sell) position.

Finally, we can also consider the returns per unit time and the realized return per unit time, these are defined by

$$
\begin{aligned}
R I_{n} & =R_{n} / n \\
R R I_{n} & =R R_{n} / n \\
R R I & =\sum_{n=2}^{\infty} R R I_{n} \operatorname{Prob}(N=n)
\end{aligned}
$$

In what follows we shall examine the statistical properties of the above returns for MA trading rules under our different distributional assumptions.

\section{Moving average rules, theory}

Our first Proposition considers the properties of $R R$ defined in (2.11) when the underlying stochastic process is Markovian, we assume that we hold whilst prices rise and sell the first time prices fall. To be specific, we buy initially for whatever reasons, if prices rise, we continue to hold and we hold until prices fall at which time we sell.

\section{Proposition 1}

For an MA(2) rule given by Equation 2.5 or 2.6 and a stationary Markovian process such as (2.3) or (2.4) the characteristic function of $R R, \phi_{\mathrm{R}}(s)$, is given by

$$
\phi_{\mathrm{R}}(s)=\left(1-\pi_{1}\right) \phi^{-}(s)+\frac{\pi_{1}\left(1-p_{11}\right) \phi^{+}(s) \phi^{+-}(s)}{1-p_{11} \phi^{++}(s)}
$$

where $\phi^{++}(s)=E\left(\exp \left(i X_{t} s\right) \mid X_{t}>0, X_{t-1}>0\right), \phi^{+-}(s)=E\left(\exp \left(i X_{t} s\right) \mid X_{t}<0, X_{t-1}>0\right), \quad \phi^{+}(s)=$ $E\left(\exp \left(i X_{t} s\right) \mid X_{t}>0\right), \quad \phi^{-}(s)=E\left(\exp \left(i X_{t} s\right) \mid X_{t}<0\right) \quad$ and $\quad p_{11}=\operatorname{prob}\left(X_{t}>0 \mid X_{t-1}>0\right), \quad \pi_{1}=$ $\operatorname{prob}\left(X_{1}>0\right)$.

Proof

$$
\begin{array}{rlrl}
\operatorname{Prob}(N=n) & =\operatorname{Prob}\left(X_{2}>0, \ldots,\right. & \left.X_{n-1}>0, X_{n}<0 \mid X_{1}>0\right) \cdot \operatorname{Prob}\left(X_{1}>0\right) \\
& =p_{11}^{n-2}\left(1-p_{11}\right) \pi_{1} & & \text { if } n \geqslant 2 \\
& =\left(1-\pi_{1}\right) & & \text { if } n=1
\end{array}
$$




$$
\begin{gathered}
\therefore \quad E\left(\exp \left(i \sum_{t=1}^{N} X_{t} s\right) / N=n\right)=\phi^{+}(s) \phi^{n-2}(s) \phi^{+-}(s) \quad \text { if } n \geqslant 2 \\
=\phi^{-}(s) \quad \text { if } n=1 \\
\therefore \quad E\left(\exp \left(i \sum_{t=1}^{N} X_{t} s\right)\right)=\sum_{n=1}^{\infty} E\left(\exp \left(i \sum_{t=1}^{N} X_{s} s\right) / N=n\right) \operatorname{Prob}(N=n)
\end{gathered}
$$

and the result follows.

\section{Comment}

The probability $\pi_{1}$ needs to be the steady state probability to guarantee that the process is Markovian and stationary. However, $\pi_{1}$ can be allowed to take other values as long as the terms $\phi^{++}(s)$ and $\phi^{+-}(s)$ stay the same. In this case $\phi^{-}(s)$ may change.

\section{Corollary (1.1)}

If we condition on the event $X_{1}>0$, which means that we know that $X_{1}>0$ prior to going long in the asset, then $\phi_{\mathrm{R} 1}(s)=\left(1-p_{11}\right) \phi^{+}(s) \phi^{+-}(s) /\left(1-p_{11} \phi^{++}(s)\right)$.

\section{Proof}

A simple proof is to put $\pi_{1}=1$ and the result follows. The substitution $\pi_{1}=1$ needs to be interpreted in the light of comments after the main proof. Note that $\phi_{\mathrm{R} 1}(s)$ is the characteristic function of $R R$ where we have decided to buy and we know that returns in the first period are positive.

\section{Corollary (1.2)}

If the $\left(X_{\mathrm{t}}\right)$ process is iid then $\phi^{++}(s)=\phi^{+}(s), \quad \phi^{+-}(s)=\phi^{-}(s) \quad$ and $\quad \phi_{\mathrm{R}}(s)=(1-$ $\left.p_{11}\right) \phi^{-}(s) \phi^{+}(s) /\left(1-p_{11} \phi^{+}(s)\right)$.

\section{Corollary (1.3)}

If, the process satisfies conditions of Corollary 1.1, and $X_{t}$ has a symmetric distribution $\phi_{\mathrm{R}}(z)=$ $\phi^{-}(s) \phi^{+}(s) /\left(2-\phi^{+}(s)\right)$ (Acar, 1993: 3.28).

\section{Proof}

Put $p_{11}=\frac{1}{2}$ in Corollary 1.1 . 
We note in passing that it does not seem possible to find a closed form solution for the distribution of $R R I$ even under the assumption that $X_{t}$ is iid. Since there is great interest in the 2 state Markovian switching model given by Equation 2.3 we shall present a detailed investigation of the properties of this model in the next section. We further remark that a contrarian strategy would lead to an analogous result to Proposition 1. Contrarian strategies, and their analysis, are of great interest in finance.

\section{Corollary (1.4)}

A contrarian strategy in Proposition 1.1 is to buy for whatever reasons, hold as long as $X_{\mathrm{i}}<0$, and sell when $X_{n}>0$, which, for a stationary Markovian framework leads to the following, define

$$
\begin{aligned}
\phi^{--}(s) & =E\left(\exp \left(i X_{t} s\right) \mid X_{t}<0, X_{t-1}<0\right) \\
\phi^{-+}(s) & =E\left(\exp \left(i X_{t} s\right) \mid X_{t-1}<0, X_{t}>0\right) \\
p_{00} & =\operatorname{Prob}\left(X_{t}<0 \mid X_{t-1}<0\right)
\end{aligned}
$$

Then $\phi_{\mathrm{R}}(s)$ is the characteristic function of $R R$ where

$$
\phi_{\mathrm{R}}(s)=\pi_{1} \phi^{+}(s)+\frac{\left(1-\pi_{1}\right)\left(1-p_{00}\right) \phi^{-}(s) \phi^{+-}(s)}{1-p_{00} \phi^{++}(s)}
$$

\section{Proof}

The proof is identical to Proposition 1.

An interesting extension to Corollary (1.3) is to ask ourselves, how does the $p d f$ of $R R$ change given the information that $X_{1}>0, X_{2}>0, \ldots X_{k}>0$ ? We shall answer this in Corollary (1.5).

\section{Corollary (1.5)}

If we have the information that $X_{1}>0, X_{2}>0, \ldots, X_{k}>0$, then $\phi_{\mathrm{R} k}(s)=\phi_{\mathrm{R} 1}(s)$ for $k=1$ (this is just a restatement of Corollary (1.1) and $\phi_{\mathrm{R} k}(s)=\phi^{+}(s) \phi^{(k+1)}(s) \phi_{\mathrm{R}}(s)$ for $k \geqslant 2$.

Proof

$$
E\left(\exp \left(i \sum_{t=1}^{N} X_{t} s\right) \mid N=n, n \geqslant k\right)=\phi^{+}(s) \phi^{(k-1)}(s) \cdot E\left(\exp \left(i \sum_{t=k+1}^{N} X_{t} s\right) \mid N=n, n \geqslant k\right)
$$

and the result follows from Proposition 1.

In the case that $X_{t}$ is (iid) we would want to know that if returns are symmetric about 0 then $E(R R)=0$, we next prove that it is true. To do so we first calculate $E(R R)$ from Proposition 1 . 


\section{Proposition 2}

For the rule and the process as described in Proposition 1

$$
E(R R)=\left(1-\pi_{1}\right) \mu_{-}+\pi_{1}\left(\mu_{+-}+\mu_{+}\right)+p_{11} \pi_{1} \mu_{++} /\left(1-p_{11}\right)
$$

where $\mu_{+}=E\left(X_{t} \mid X_{t}>0\right), \mu_{-}=E\left(X_{t} \mid X_{t}<0\right), \mu_{+-}=E\left(X_{t} \mid X_{t}<0, X_{t-1}>0\right)$ etc.

\section{Proof}

Differentiating the characteristic function gives

$$
\begin{gathered}
\frac{\partial \phi_{\mathrm{R}}(s)}{\partial s}=\left(1-\pi_{1}\right) \frac{\partial \phi^{-}(s)}{\partial s} \\
+\frac{\pi_{1}\left(1-p_{11}\right)\left[\left(1-p_{11} \phi^{++}(s)\right)\left(\frac{\partial \phi^{+-}(s)}{\partial s} \phi^{+}(s)+\frac{\partial \phi^{+}(s)}{\partial s} \phi^{+-}(s)\right)-p_{11} \frac{\partial \phi^{++}(s)}{\partial s}\left(\phi^{+}(s) \phi^{+-}(s)\right)\right]}{\left(1-p_{11} \phi^{++}(s)\right)^{2}}
\end{gathered}
$$

Using $\phi_{\mathrm{R}}^{\prime}(0)=i E(R R), \phi^{\prime+}(0)=i \mu_{+}$etc and $\phi^{+}(0)=\phi^{-}(0)=\phi^{++}(0)=1$ etc gives the result.

This has the implication that if returns are symmetric and iid then $\pi_{1}=p_{11}=\frac{1}{2}, \mu^{+}=-\mu^{-}$, $\mu^{++}=\mu_{+}$and $\mu_{+-}=\mu_{-}$, so that, as anticipated, $E(R R)=0$.

\subsection{Moving average trading rules and the Markovian switching model}

We now consider Proposition 1 under the assumption that $X_{\mathrm{t}}$ is generated by

$$
X_{t}=\alpha_{0}\left(1-S_{t}\right)+\alpha_{1} S_{t}+\left(\sigma_{0}\left(1-S_{t}\right)+\sigma_{1} S_{\mathrm{t}}\right) e_{t}
$$

The above conditions imply that $p d f\left(X_{t} \mid S_{t}=1\right) \stackrel{d}{\sim} N\left(\alpha_{1}, \sigma_{1}^{2}\right), p d f\left(X_{t} \mid S_{t}=0\right) \stackrel{d}{\sim} N\left(\alpha_{0}, \sigma_{0}^{2}\right)$, where $\stackrel{d}{\sim} N\left(\mu, \sigma^{2}\right)$ means distributed as a normal with mean $\mu$ and variance $\sigma^{2}$. Furthermore, under the stationary assumption, $\pi=\operatorname{Prob}\left(S_{t}=1\right)$, the unconditional probability,

$$
p d f\left(X_{t}\right) \stackrel{d}{\sim}(1-\pi) N\left(\alpha_{0}, \sigma_{0}^{2}\right)+\pi N\left(\alpha_{1}, \sigma_{1}^{2}\right)
$$

This tells us that $X_{t}$ is distributed unconditionally as a mixture of normals. We now consider the characteristic function of $X_{t}$ given $X_{t}>0$, we present the result which is probably well-known as Lemma 1.

\section{Lemma 1}

If $y \stackrel{d}{\sim} N\left(\mu, \sigma^{2}\right)$ then 


$$
\begin{aligned}
\phi^{+}(s) & =E(\exp (i s y) \mid y>0) \\
& =\frac{\exp \left[\frac{\left(\mu+\sigma^{2} i s\right)^{2}-\mu^{2}}{2 \sigma^{2}}\right] \Phi\left(\frac{\mu+\sigma^{2} i s}{\sigma}\right)}{\Phi\left(\frac{\mu}{\sigma}\right)}
\end{aligned}
$$

and

$$
\phi^{-}(s)=E(\exp (i s y) / y<0)=\phi^{+}(-s) \text { if } \mu=0
$$

$\Phi$ is the standardized normal distribution function.

\section{Proof of Lemma 1}

Let

$$
\begin{aligned}
m^{+}(s) & =E(\exp (s y) \mid y>0) \\
& =\frac{\int_{0}^{\infty} \exp (s y) \frac{1}{\left(2 \pi \sigma^{2}\right)^{1 / 2}} \exp \left(\frac{-(y-\mu)^{2}}{2 \sigma^{2}}\right) \mathrm{d} y}{\Phi(\mu / \sigma)}
\end{aligned}
$$

Completing the square and some boring algebra gives us the result, note that $\phi^{+}(s)=m^{+}(i s)$.

We now note that

$$
\phi^{+}(s)=E\left(\exp \left(i X_{t} s\right) \mid X_{t}>0\right)
$$

where the $p d f$ of $X_{t}$ is given by (3.2)

$$
\begin{aligned}
\phi^{+}(s) & =\frac{(1-\pi) \int_{0}^{\infty} \mathrm{e}^{i X_{t} s} N\left(\alpha_{0}, \sigma_{0}^{2}\right) \mathrm{d} X_{t}+\pi \int_{0}^{\infty} \mathrm{e}^{i X_{t} s} N\left(\alpha_{1}, \sigma_{1}^{2}\right) \mathrm{d} X_{t}}{(1-\pi) \Phi\left(\frac{\alpha_{0}}{\sigma_{0}}\right)+\pi \Phi\left(\frac{\alpha_{1}}{\sigma_{1}}\right)} \\
& =\frac{\left[(1-\pi) \exp \left[\frac{\left(\alpha_{0}+\sigma_{0}^{2} i s\right)^{2}-\alpha_{0}^{2}}{2 \sigma_{0}^{2}}\right] \Phi\left(\frac{\alpha_{0}+\sigma_{0}^{2} i s}{\sigma_{0}}\right)+\pi \exp \left[\frac{\left(\alpha_{1}+\sigma_{1}^{2} i s\right)^{2}-\alpha_{1}^{2}}{2 \sigma_{1}^{2}}\right] \Phi\left(\frac{\alpha_{1}+\sigma_{1}^{2} i s}{\sigma_{1}}\right)\right]}{\left((1-\pi) \Phi\left(\frac{\alpha_{0}}{\sigma_{0}}\right)+\pi \Phi\left(\frac{\alpha_{1}}{\sigma_{1}}\right)\right)}
\end{aligned}
$$

Using Lemma 1, we can now prove Proposition 3 which details the characteristic function $\phi_{\mathrm{R}}(s)$ described in Proposition 1 using the model in Equation 3.1. Lemma 1 can be used directly to evaluate $\phi^{+}(s)$. It is straightforward to calculate $\phi^{-}(s)$ if we know $\phi^{+}(s)$.

We define the following, $\pi_{i j}=\operatorname{Prob}\left(X_{t-1}=i, X_{t}=j\right), i, j=0,1$. 


\section{Proposition 3}

For the model given by Equation 3.1 the following expressions hold

$$
\phi^{++}(s)=\frac{\sum_{k=0}^{1} \sum_{j=0}^{1} \pi_{k j} \Phi\left(\frac{\alpha_{k}}{\sigma_{k}}\right) \exp \left(\frac{\left(\alpha_{j}+\sigma_{j}^{2} i s\right)^{2}-\alpha_{j}^{2}}{2 \sigma_{j}^{2}}\right) \Phi\left(\frac{\alpha_{j}+i \sigma_{j}^{2} s}{\sigma_{j}}\right)}{\sum_{k=0}^{1} \sum_{j=0}^{1} \pi_{k j} \Phi\left(\frac{\alpha_{k}}{\sigma_{k}}\right) \Phi\left(\frac{\alpha_{j}}{\sigma_{j}}\right)}
$$

and

$$
\phi^{+-}(s)=\frac{\sum_{k=0}^{1} \sum_{j=0}^{1} \pi_{k j} \Phi\left(\frac{\alpha_{k}}{\sigma_{k}}\right) \exp \left(\frac{\left(\alpha_{j}+i s \sigma_{j}^{2}\right)^{2}-\alpha_{j}^{2}}{2 \sigma_{j}^{2}}\right)\left(1-\Phi\left(\frac{\alpha_{j}+i \sigma_{j}^{2} s}{\sigma_{j}}\right)\right)}{\sum_{k=0}^{1} \sum_{j=0}^{1} \pi_{k j} \Phi\left(\frac{\alpha_{k}}{\sigma_{k}}\right)\left(1-\Phi\left(\frac{\alpha_{j}}{\sigma_{j}}\right)\right)}
$$

\section{Proof}

See Appendix.

The conclusion of Lemma 1 and Proposition 2 allows us to fully evaluate $\phi_{\mathrm{R}}(s)$ for Equation 3.1 described in Proposition 1. In turn we can use this result to evaluate the distribution function via numerical integration. We shall illustrate our discussion and results by a worked example.

\section{Moving average rules, application}

\subsection{An application to the FTSE Futures Contract}

The purpose of this section is to show that we can gain insight on the empirical returns generated by the moving average of order 2 using the theoretical results established in previous sections. Our methodology is straightforward. This consists of

(a) establishing the Markovian process which generates the underlying financial asset

(b) observing the distributional properties of the returns generated by the $S(2)$ rule

(c) comparing the properties of the $S(2)$ empirical returns with their theoretical values assuming that the financial asset follows a Markovian process with known parameters.

The most obvious choice of financial instrument is the FTSE futures contract. Previous researchers (Knight et al., 1995) have shown that FTSE Futures returns are not particular normal nor are they Markovian over a different period than the one we consider. Bearing this in mind we shall fit the distribution more as a demonstration of the method than as a serious investigation of the process, which would require much more careful analysis. Our simulations roll forward each futures contract as it 
approaches the settlement data, just as a futures trader would. The futures contracts are the first future contract until the before last trading day. For all futures contracts, a unique time series of logarithmic returns has been constructed as $X_{t}=\operatorname{Ln}\left(P_{t} / P_{t-1}\right)$ from May 1984 to September 1995 included. Table 1 illustrates an example of rollovers. Characteristics of the underlying time series can be found in Table 2, whereas estimates of the Markovian model are given in Table 3.

The Markov switching model fits two different regimes: in the first regime we have a large negative mean, insignificantly different from zero, and high volatility, in the second regime the mean is positive but with lower volatility. The values in Table 3 could be used to calculate $\pi_{1}$ and $\pi_{i j}$, however it is much simpler to calculate these directly from the data.

In Figure 1 we present the filtered probabilities of being in the first regime, these tell us when the probability of being in the first regime is high/low given all the sample information and correspond to a plot of a regression line in the case where the variable $S_{t}$ is as in (2.3). In particular, the three high peaks near 800, 1600 and 2000 correspond to the October 87 crash, the August 90 invasion of Kuwait and the September 92 withdrawal from the ERM. Overall we have a high probability of being in the second regime, i.e. $\pi=0.96$, that is reflected also by the transition probabilities: the probability of remaining in the second regime is 0.994 , the positive mean regime is highly persistent whilst the negative mean regime is not, the probability of remaining on the first regime is 0.858 . Considering the realized returns of a MA(2) rule given by Equations 2.5-2.6 we have 1730 observations over the sample of 2883.

Table 1. Futures time series

\begin{tabular}{lllll}
\hline Date & $\begin{array}{l}\text { Delivery } \\
\text { month }\end{array}$ & $\begin{array}{l}\text { Price (March 95) } \\
P_{1,1}\end{array}$ & $\begin{array}{l}\text { Price (June 95) } \\
P_{2,1}\end{array}$ & $\begin{array}{l}\text { Logarithmic returns } \\
X_{t}=\operatorname{Ln}\left(P_{t} / P_{t-1}\right)\end{array}$ \\
\hline 14 Mar 95 & Mar 95 & 3057 & - & - \\
15 Mar 95 & Mar 95 & 3045 & - & $-0.39 \%=\operatorname{Ln}(3045 / 3057)$ \\
16 Mar 95 & Mar 95 & 3093 & 3099.5 & $1.56 \%=\operatorname{Ln}(3093 / 3045)$ \\
17 Mar 95 & Jun 95 & - & 3090.0 & $-0.31 \%=\operatorname{Ln}(3090 / 3099.5)$ \\
20 Mar 95 & Jun 95 & - & 3142.0 & $1.67=\operatorname{Ln}(3142 / 3090)$ \\
21 Mar 95 & Jun 95 & - & 3158.0 & $0.51 \%=\operatorname{Ln}(3158 / 3142)$ \\
\hline
\end{tabular}

Table 2. FTSE futures basic statistics

\begin{tabular}{lc}
\hline & Underlying returns \\
\hline Mean & $0.0191 \%$ \\
Standard deviation & $1.0781 \%$ \\
Sample variance & $0.0116 \%$ \\
Kurtosis & 25.657 \\
Skewness & -1.665 \\
Range & $24.81 \%$ \\
Minimum & $-16.72 \%$ \\
Maximum & $8.09 \%$ \\
Count & 2883 \\
\hline
\end{tabular}


Table 3. Markovian model

\begin{tabular}{lc}
\hline Variable & Value and \\
\hline$\alpha_{0}$ & -0.003142122 \\
& $(0.003044925)$ \\
$\alpha_{1}$ & 0.000322833 \\
& $(0.000180365)$ \\
$\sigma_{0}^{2}$ & 0.0010245 \\
& $(0.0002009)$ \\
$\sigma_{1}^{2}$ & 0.00008003 \\
& $(0.00000025)$ \\
$q$ & 0.99447367 \\
& $(0.00197927)$ \\
$p$ & 0.858185885 \\
& $(0.050910174)$ \\
\hline
\end{tabular}

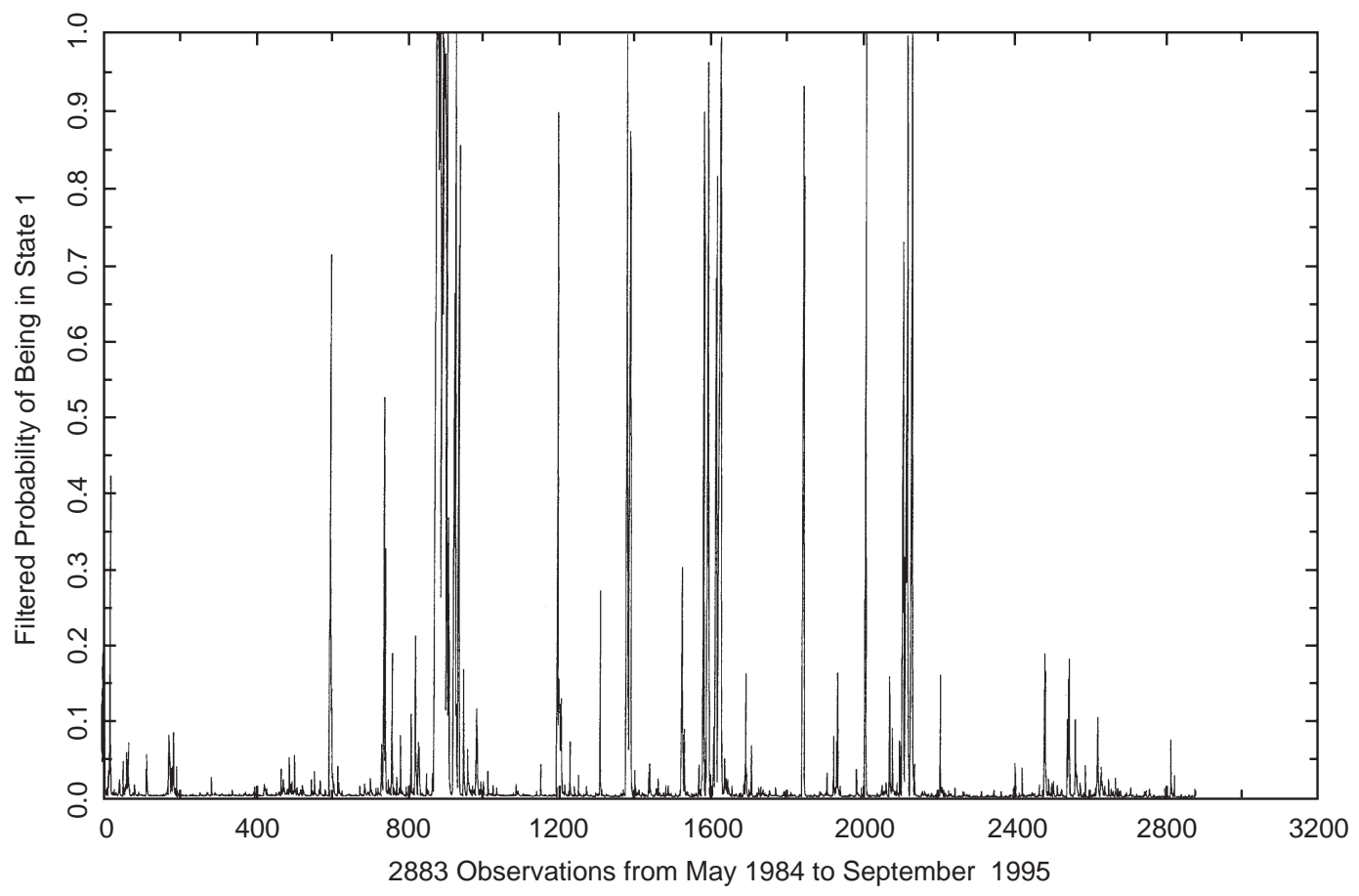

Fig. 1. FT100 futures and 2 state Markov model, probability of state 1. 
We next report the first moments in Proposition 2 calculated by the characteristic function of RR for an $\mathrm{MA}(2)$ rule given by (2.5-2.6) and a stationary Markovian process of Table 3. We have $\mu^{-}=\phi^{\prime}-(0)=-0.00779, \quad \mu^{+}=\phi^{\prime+}(0)=0.00785, \quad \mu^{+-}=\phi^{++}(0)=-0.01070 \quad$ and $\quad \mu^{++}=$ $\phi^{\prime++}(0)=0.00995$, these should be compared with the empirical moments that are respectively $-0.00804,0.00775,-0.00788$ and 0.00778 . We see that the Markovian model does a reasonable job in replicating the sign and level of the returns generated by an MA of order 2.

The relevant question is when will a rule such as the one we have analysed in the previous section be profitable. Turning to the general case, it has been shown by Silber (1994) and others that MA rules are profitable when there is mean reversion about a positive trend in prices. The rule's ability to let us out of the market when below the trend may allow us to outperform a buy and hold strategy. Turning to the MA(2) model with Markovian returns in particular we can use the results of proposition 2 to infer circumstances when we might make positive realized returns. If we take the simplifying case that $\pi_{1}=1 / 2$, i.e. that on average half of the returns are positive then $E(R R)$ can be simplified to $E(R R)=1 / 2\left(\mu^{-}+\mu^{+-}+\mu^{+}+\left(p_{11} /\left(1-p_{11}\right)\right) \mu^{++}\right.$. A set of conditions that would guarantee a positive expected return could be $\pi_{1}=1 / 2, \mu^{+}>-\mu^{-}, \mu_{++}>-\mu^{+-}$and $p_{11}>1 / 2$. In words these conditions say that if returns are positive this period they are more likely to be positive next period, if upside returns are on average higher than downside returns next period given positive returns this period then we make a profit on average. Broadly, these conditions point to two requirements for Markovian processes to lead to profitable trading. These are the presence of some positive autocorrelation and higher upside returns than downside returns. We present a lemma which gives further light on these conditions.

\section{Lemma 2}

If $\alpha_{0}$ is not equal to $\alpha_{1}$, then $\operatorname{Corr}\left(X_{t}, X_{t-1}\right)>0$ iff $p_{11}>\pi_{1}$.

\section{Proof}

We show in the appendix that

$$
\operatorname{Corr}\left(X_{t}, X_{t-1}\right)=\frac{\left(\alpha_{0}-\alpha_{1}\right)^{2}\left(p_{11}-\pi_{1}\right)}{\left(\alpha_{0}-\alpha_{1}\right)^{2}(1-\pi)+\left(\sigma_{0}^{2}(1-\pi)+\sigma_{1}^{2} \pi\right)}
$$

This lemma tells us that $\operatorname{Corr}\left(X_{t}, X_{t-1}\right)$ is positive iff the probability that $X_{t}>0$ given $X_{t-1}>0$ is greater than the probability that $X_{t}>0$, and that the returns in the two regimes are different. Intuitively this is the condition that will reward our trading rule. The positive autocorrelation gives us the mean reversion and $\mu^{+}>-\mu^{-}$and $\mu^{++}>-\mu^{+-}$correspond to a positive trend.

\section{Conclusions}

In this article we have presented a taxonomy of trading rules and returns. In one particular case, the $\mathrm{MA}(2)$, it is possible to compute the characteristic function, and hence the distribution function, of 
realized returns. These results are refined in order to take account of partial information. Finally the calculations necessary to use these results in the case of the Gaussian Markov switching regime model are presented and an empirical example is shown. It becomes clear that the analysis produces a large number of interesting statistics that could be used to analyse not just the trading rule, but, possibly more importantly, the properties of the underlying price process itself. A full empirical analysis would involve a separate paper which we plan to do in the future. Further extensions to our work that we hope to consider involve discussion of $\mathrm{MA}(\infty)$ rules and other distributional assumptions governing returns.

\section{References}

Acar, E. (1993) Economic evaluation of financial forecasting, PhD Thesis, City University, London.

Alexander, S. (1961) Price movements in speculative markets: trends or random walks, Industrial Management Review, 2, 7-26.

Blume, L., Easley, D. and O'Hara, M. (1994) Market statistics and technical analysis: the role of volume, Journal of Finance, 49, 153-81.

Brock, W., Lakonishok, J. and LeBaron, B. (1992) Simple technical rules and the stochastic properties of stock returns, Journal of Finance, 47, 1731-64.

Corrado, C.J. and Lee, S.H. (1992) Filter rule tests of the economic significance of serial dependencies in daily stock returns, Journal of Financial Research, 15(4), 369-87.

Donchian, R.D. (1957) Trends following methods in commodity analysis, Commodity Year Book 1957.

Goodhart, C.A.E. and Curcio, R. (1992) When support/resistance levels are broken, can profits be made? Evidence from the foreign exchange market, LSE Financial Markets Group Discussion Paper Series, L. 142, July.

Hamilton, J.D. (1989) A new approach to the economic analysis of nonstationary time series and the business cycle, Econometrica, 57, 357-84.

Knight, J. Satchell, S. and K. Tran (1995) Statistical modelling of asymmetric risk in asset returns, Applied Mathematical Finance, 1(2), 155-72.

LeBaron, B. (1991) Technical trading rules and regime shifts in foreign exchange, University of Wisconsin, Social Science Research, Working Paper 9118.

LeBaron, B. (1992) Do moving average trading rule results imply nonlinearities in foreign exchange markets, University of Wisconsin, Social Science Research, Working Paper 9222.

Levich, R.M. and Thomas, L.R. (1993) The significance of technical trading-rule profits in the foreign exchange market: a bootstrap approach, Journal of International Money and Finance, 12, 451-74.

Leuthold, R.M., Garcia, P. and Lu, R. (1994) The returns and forecasting ability of large traders in the frozen pork bellies futures market, Journal of Business, 67(3), 459-71.

Neftci, S.N. (1991) Naive trading rules in financial markets and Wiener-Kolmogorov prediction theory: a study of 'technical analysis', Journal of Business, 64, 549-71.

Silber, W. (1994) Technical trading: when it works and when it doesn't, Journal of Derivatives, 1, Spring, 3944.

Taylor, S.J. (1994) Trading futures using the channel rule: a study of the predictive power of technical analysis with currency examples, Journal of Futures Markets, 14(2), 215-35. 


\section{Appendix}

Derivation of the characteristic functions $\phi^{+-}(s)$ and $\phi^{++}(s)$.

\section{Proof}

We write out

$$
X_{t}=\alpha_{0}\left(1-S_{t}\right)+\alpha_{1} S_{t}+\left(\sigma_{0}\left(1-S_{t}\right)+\sigma_{1} S_{t}\right) e_{t}
$$

Let $i$ refer to state of $S_{t-1}, i=0,1$, and $j$ refer to state of $S_{t}$. Prob $\left(S_{t-1}=k, S_{t}=j\right)=\pi_{k j}$, in particular, letting $p_{11}=p$ and $p_{00}=q, \pi_{01}=(1-q)(1-\pi), \pi_{00}=q(1-\pi), \pi_{11}=p \pi, \pi_{10}=(1-p) \pi$.

The joint $p d f\left(X_{t}, X_{t-1} \mid S_{t-1}=k, S_{t}=j\right)=N\left(\alpha_{k}, \sigma_{k}^{2}\right) N\left(\alpha_{j}, \sigma_{j}^{2}\right)$ where, for example, $p d f\left(X_{t}\right.$, $\left.X_{t-1} \mid S_{t-1}=1, S_{t}=0\right)=N\left(\alpha_{1}, \sigma_{1}^{2}\right) N\left(\alpha_{0}, \sigma_{0}^{2}\right)$. Thus, by the law of total probability $p d f\left(X_{t}, X_{t-1}\right)=$ $\sum_{k=0}^{1} \sum_{j=0}^{1} \pi_{k j} N\left(\alpha_{k}, \sigma_{k}^{2}\right) N\left(\alpha_{j}, \sigma_{j}^{2}\right)$ and $\operatorname{Prob}\left(X_{t}>0, X_{t-1}>0\right)=\sum \sum \pi_{k j} \int_{0}^{\infty} \int_{0}^{\infty} N\left(\alpha_{k}, \sigma_{k}^{2}\right) N\left(\alpha_{j}\right.$, $\left.\sigma_{j}^{2}\right) \mathrm{d} X_{t} \mathrm{~d} X_{t-1}=\sum \sum \pi_{k j} \Phi\left(\alpha_{k} / \sigma_{k}\right) \Phi\left(\alpha_{j} / \sigma_{j}\right)$. By repeated use of the calculations in Lemma 1,

$$
P d f\left(X_{t}, X_{t-1} \mid X_{t}>0, X_{t-1}>0\right)=\frac{\sum \sum \pi_{k j} N\left(\alpha_{k}, \sigma_{k}^{2}\right) N\left(\alpha_{j}, \sigma_{j}^{2}\right)}{\sum \sum \pi_{k j} \Phi\left(\frac{\alpha_{k}}{\sigma_{k}}\right) \Phi\left(\frac{\alpha_{j}}{\sigma_{j}}\right)}
$$

for $X_{t}>0, X_{t-1}>0$.

Finally

$$
E\left(\exp \left(i X_{t} s\right) \mid X_{t}>0, X_{t-1}>0\right)=\frac{\sum \sum \pi_{k j} \Phi\left(\frac{\alpha_{k}}{\sigma_{k}}\right) \exp \left(\frac{\left(\alpha_{j}+\sigma_{j}^{2} i s\right)^{2}-\alpha_{j}^{2}}{2 \sigma_{j}^{2}}\right) \Phi\left(\frac{\alpha_{j}+i \sigma_{j}^{2} s}{\sigma_{j}}\right)}{\sum \sum \pi_{k j} \Phi\left(\frac{\alpha_{k}}{\sigma_{k}}\right) \Phi\left(\frac{\alpha_{j}}{\sigma_{j}}\right)}
$$

Similar calculations apply for $\phi_{+-}(s)$ except that for $X_{t}, X_{t}<0$

$$
\operatorname{Prob}\left(X_{t}<0, X_{t-1}>0\right)=\sum \sum \pi_{k j} \Phi\left(\alpha_{k} / \sigma_{k}\right)\left(1-\Phi\left(\alpha_{j} / \sigma_{j}\right)\right)
$$

$$
\begin{aligned}
E\left(\exp \left(i X_{t} s\right) \mid X_{t}<0, X_{t-1}>0\right) & =\phi^{+-}(s)=\phi^{++}(-s) \\
& =\frac{\sum_{k} \sum_{j} \pi_{k j} \Phi\left(\frac{\alpha_{k}}{\sigma_{k}}\right) \exp \left(\frac{\left(\alpha_{j}+\sigma_{j}^{2} i s\right)^{2}-\alpha_{j}}{2 \sigma_{j}^{2}}\right)\left(1-\Phi\left(\frac{\alpha_{j}+i \sigma_{j}^{2} s}{\sigma_{j}}\right)\right)}{\sum_{k} \sum_{j} \pi_{k j} \Phi\left(\frac{\alpha_{k}}{\sigma_{k}}\right)\left(1-\Phi\left(\frac{\alpha_{j}}{\sigma_{j}}\right)\right)}
\end{aligned}
$$




\section{Proof of Lemma 2}

Since

$$
\begin{aligned}
X_{t} & =\alpha_{0}\left(1-S_{t}\right)+\alpha_{1} S_{t}+\left(\sigma_{0}\left(1-S_{t}\right)+\sigma_{1} S_{t}\right) e_{t} \\
E\left(X_{t}\right) & =\alpha_{0}(1-\pi)+\alpha_{1} \pi \\
E\left(X_{t}^{2}\right) & =\alpha_{0}^{2}(1-\pi)+\alpha_{1}^{2} \pi+\left(\sigma_{0}^{2}(1-\pi)+\sigma_{1}^{2} \pi\right)
\end{aligned}
$$

and

$$
\begin{aligned}
V\left(X_{t}\right) & =\left(\alpha_{0}-\alpha_{1}\right)^{2}(1-\pi) \pi+\left(\sigma_{0}^{2}(1-\pi)+\sigma_{1}^{2} \pi\right) \\
\operatorname{Cov}\left(X_{t} X_{t-1}\right) & =E\left(X_{t} X_{t-1}\right)-E^{2}\left(X_{t}\right)
\end{aligned}
$$

and

$$
\begin{aligned}
& E\left(X_{t} X_{t-1}\right)=\alpha_{0}^{2}\left(1-2 \pi+\pi p_{11}\right)+2 \alpha_{0} \alpha_{1} \pi\left(1-p_{11}\right)+\alpha_{1}^{2} \pi p_{11} \\
& \operatorname{Corr}\left(X_{t}, X_{t-1}\right)=\frac{\left(\alpha_{0}-\alpha_{1}\right)^{2}\left(p_{11}-\pi\right)}{\left(\alpha_{0}-\alpha_{1}\right)^{2}(1-\pi) \pi+\left(\sigma_{0}^{2}(1-\pi)+\sigma_{1}^{2} \pi\right)}
\end{aligned}
$$

Result follows on inspection of (A.1). 